\title{
Coronary Heart Disease Prediction and Prevention
}

\section{Dae Hyun Kim, MD, MPH, Andrew N. Lee, MD}

\section{Introduction}

Prediction of coronary heart disease (CHD) is based on multivariable risk equations developed from population-based observational studies in which people without clinical CHD at the initiation of study were examined and followed until their first CHD events. The risk equations from the Framingham Heart Study have been widely used in our clinical practice1-3 and research. ${ }^{4,5}$ The recent report of the third National Cholesterol Education Program-Adult Treatment Panel (NCEP-ATP) incorporated the Framingham risk equations to predict ten-year absolute CHD risk and to identify certain patients who are at high risk and more likely to benefit from primary prevention with aggressive lipid-lowering treatment. ${ }^{1}$ In addition to $\mathrm{CHD}$ prediction, population-based observational studies also provide the clue to understand how much of CHD can be prevented by modifying major cardiovascular risk factors such as serum cholesterol level, blood pressure level, and current smoking. ${ }^{4,6,7}$

In this narrative review, we described how CHD prediction works and how it can be improved by including nontraditional cardiovascular risk factors. We also discussed about how likely it is to prevent the majority of CHD.

\section{How Do We Predict CHD?}

The Framingham Heart Study has developed mathematical functions to assess the relative importance of CHD risk factors and to quantify absolute CHD risk for individual patients. ${ }^{8-10}$ Detailed methods of derivation of CHD risk equations were described elsewhere. ${ }^{8}$ Briefly, sex-specific CHD risk equations were derived from a population-based sample of 2489 men and 2856 women, 30 to 74 years of age, who were free of overt cardiovascular disease at the time of their 11 th examination of the original Framingham Cohort or the initial examination of the Framingham Offspring Study in 1971 to 1974 . CHD risk factors were routinely and systematically measured during these examinations and twelve-year follow-up was obtained for the development of "hard" CHD events, defined as myocardial infarction and CHD death. Sex-specific Cox proportional hazards regression was performed to calculate the relative importance of CHD risk factors using age, current smoking, presence of diabetes, the fifth Joint National Committee on Hypertension blood pressure categories, and the second NCEP-ATP cholesterol categories as covariates. (Table 1) In addition, score sheets were developed from the beta-coefficients of Cox proportional hazards models to provide ten-year absolute CHD risk and to make it easy to implement as part of a screening program. They were adopted by the NCEP-ATP III guideline. ${ }^{1}$ (Figure 1) The predictive capability of the model using a continuous variable or a categorical variable for cholesterol level was almost identical. ${ }^{8}$ The equation is particularly useful when there are multiple mild abnormalities that increase CHD risk synergistically.

In the Framingham risk equation, several candidate risk factors such as family history of CHD, elevated fibrinogen levels, left ventricular hypertrophy on the electrocardiogram, postmenopausal estrogen replacement therapy, physical activity, high serum triglyceride, and body mass index, were not included for practical reasons, although they may contribute to the risk of CHD. ${ }^{8}$
Table 1. Multivariate-Adjusted Relative Risks (RR) for CHD from the Framingham Heart Study: Twelve-Year Follow-Up of 2489 Men and 2856 Women (Adapted from Wilson et a $\left.\right|^{8}$ )

\begin{tabular}{lllll}
\hline & Men & \multicolumn{3}{l}{ Women } \\
\hline Risk Factors & RR & $95 \% \mathrm{Cl}$ & RR & $95 \% \mathrm{Cl}$ \\
\hline Age (Years) & $1.05 \ddagger$ & $1.04-1.06$ & $1.04 \ddagger$ & $1.03-1.06$ \\
\hline Blood Pressure & & & & \\
\hline Normal & 1.00 & Referent & 1.00 & Referent \\
\hline High Normal & 1.32 & $0.98-1.78$ & 1.34 & $0.88-2.05$ \\
\hline Hypertension Stage I & $1.73 \ddagger$ & $1.32-2.26$ & $1.75 \dagger$ & $1.21-2.54$ \\
\hline Hypertension Stage II & $1.92 \ddagger$ & $1.42-2.59$ & $2.19 \ddagger$ & $1.46-3.27$ \\
\hline Cigarette Use (Yes/No) & $1.71 \ddagger$ & $1.39-2.10$ & $1.49 \dagger$ & $1.13-1.97$ \\
\hline Diabetes (Yes/No) & $1.47^{*}$ & $1.04-2.08$ & $1.80 \dagger$ & $1.18-2.74$ \\
\hline LDL Cholesterol (mg/dL) & & & & \\
\hline$<130$ & 1.00 & Referent & 1.00 & Referent \\
\hline $130-159$ & 1.19 & $0.91-1.54$ & 1.24 & $0.84-1.81$ \\
\hline$\geq 160$ & $1.74 \ddagger$ & $1.36-2.24$ & $1.68 \dagger$ & $1.17-2.40$ \\
\hline HDL Cholesterol (mg/dL) & & & & \\
\hline$<35$ & $1.46 \dagger$ & $1.15-1.85$ & $2.08 \dagger$ & $1.33-3.25$ \\
\hline $35-59$ & 1.00 & Referent & 1.00 & Referent \\
\hline$\geq 60$ & $0.61^{*}$ & $0.41-0.91$ & $0.64 \dagger$ & $0.47-0.87$ \\
\hline
\end{tabular}

$* 0.01<P<0.05, \dagger 0.001<P<0.01, \ddagger P<0.001$.

\section{How Well Does CHD Prediction Equation Work?}

The performance of the CHD risk prediction models has been examined according to discrimination and calibration. ${ }^{4.5}$ From the coefficients of the prediction model, a risk score can be calculated for each person by multiplying the person's risk factor level by the associated coefficient for that risk variable, then summing all these products. Those with a higher risk score from a prediction model are expected to have higher CHD events. Discrimination is the ability of a predictive model to separate those who experience hard CHD events from those who do not. It can be quantified by c-statistic or the area under a receiver operating characteristic (ROC) curve (AUC) which indicates the probability that a person who had an incident CHD event within a specified time had a higher risk score than a person who did not have an event by that time. ${ }^{11,12}$ The AUC has a range between 0.5 and 1 . When the variables in the prediction model are unrelated to the event, the expected AUC would be 0.5 . The greater the AUC is, the better the prediction model works. Calibration, another measure of performance of the prediction model, measures how closely predicted outcomes agree with actual outcomes. 
Table B1. Estimate of 10-Year Risk for Men (Framingham Point Scores)
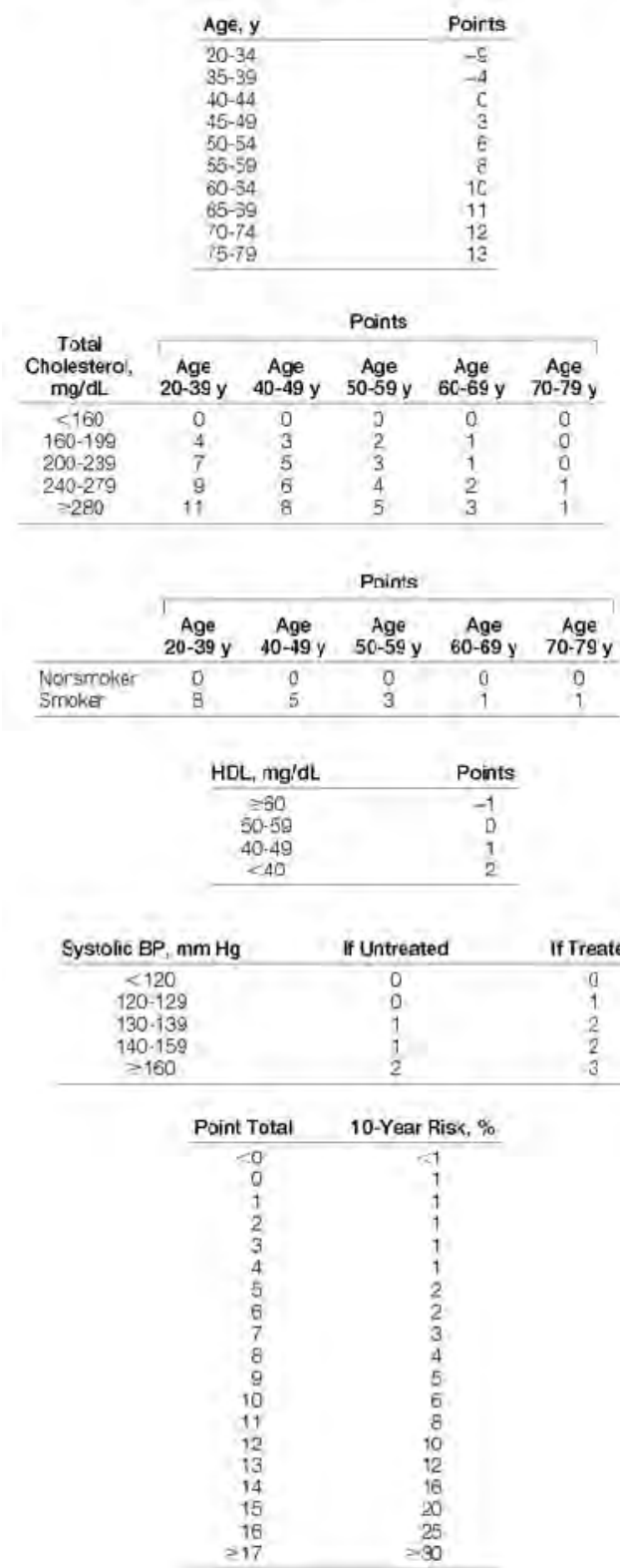

Table B2 Estimate of 10-Year Risk for Women (Framingham Point Scores)
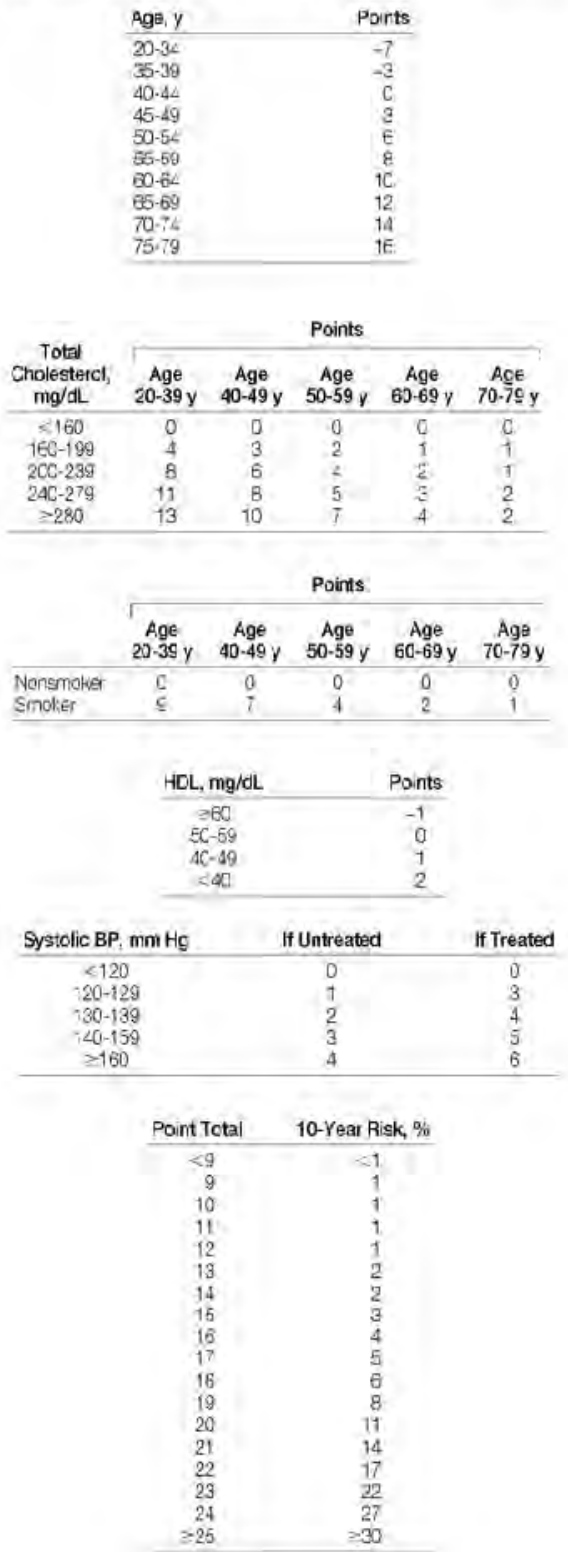

Figure 1. Score Sheets for Estimation of Ten-Year CHD Risk for Men and Women (Adapted from NCEP-ATP III Guideline')

Since the Framingham risk equation was derived from a community sample of white middle-class individuals from a suburb of Boston, concerns have existed regarding its generalizability to other populations. ${ }^{8}$ To test the validity, the sex-specific Framingham risk equations were applied to six prospectively studied, ethnically diverse cohorts including the Atherosclerosis Risk in Communities Study (ARIC), Physicians' Health Study, Honolulu Heart Program, Puerto Rico Heart Health Program, Strong Heart Study, and the Cardiovascular Health Study. ${ }^{5}$ The performance of the predication equation was compared using discrimination and calibration. For white men and women and for black men and women, the Framingham risk equations performed reasonably well within five years of follow-up. (Figure 2) Among Japanese American and Hispanic men and Native American women, the equation overestimated the risk. However, after taking into account different prevalence of risk factors and underlying $\mathrm{CHD}$ rates, it performed well in these populations.

Then, how much of incident CHD events are explained by known risk factors? The CHD prediction equations have been used to answer this question. In the Seven Countries Study, the prediction equation using systolic blood pressure, cholesterol, smoking, and age derived from the US railroad workers was applied to men in 
five European countries. ${ }^{13}$ Fifty one percent of CHD events occurred in top quintile of the predicted CHD risk. Based on this finding, it is often said that only 50 percent of the CHD is explained by the known risk factors. In another study, the Framingham equation was applied to the National Health and Nutrition Examination Survey I, a probability sample of the US population. ${ }^{14}$ Thirty nine percent of CHD deaths in men and 59 percent in women occurred in top quintile of the predicted risk. Based on these findings, can we say that approximately 40 to 60 percent of CHD mortality is due to known causes and the remaining percent is due to unknown causes? This is a very common misinterpretation. Counting cases attributable to the upper end of the risk distribution defined by an arbitrarily high cut point on the scale is misleading, because most of the risk factors have a continuous graded relationship to risk. ${ }^{15}$

In a study using five large cohorts of young and middle-aged adults in the Multiple Risk Factor Intervention Trial (MRFIT) and Chicago Heart Association Detection Project in Industry (CHA) who were free of diabetes and myocardial infarction, low-risk participants who had cholesterol level less than $200 \mathrm{mg} / \mathrm{dL}$, blood pressure less than or equal to $120 / 80 \mathrm{mmHg}$, and no current smoking, experienced significantly and markedly lower CHD death rates by 77 to 92 percent than the rest of the cohorts during the mean follow-up period of 16 years for MRFIT and 22 years for $\mathrm{CHA}{ }^{6}$ This suggests that three major risk factors - serum cholesterol level, blood pressure, and smoking - account for the majority of CHD death. Similar results were found in the ARIC study. ${ }^{4}$ Not being in the bottom decile of risk score derived from traditional risk factors accounted for 72-75 percent of CHD risk in men and 89-93 percent in women. Evidences from numerous cohort studies with long follow-up have shown that the major established risk factors explain at least $75 \%$ of the CHD events within populations and there is no evidence supporting the only-50-percent claim.

\section{Can We Improve CHD Risk Equation?}

If traditional risk factors in the Framingham risk equation - total cholesterol, HDL cholesterol, current smoking, diabetes, and blood pressure - can explain 75 percent of incident CHD events,

Table 2. Area Under the ROC Curves Comparing Basic and Full Models (Adapted and modified from Chambless at $\left.\mathrm{el}^{4}\right)^{*}$

\begin{tabular}{lllll}
\hline & Black Women & White Women & Black Men & White Men \\
\hline Model & $\mathrm{N}=1798 ; \mathrm{n}=90$ & $\mathrm{~N}=5006 ; \mathrm{n}=198$ & $\mathrm{~N}=1102 ; \mathrm{n}=101$ & $\mathrm{~N}=4082 ; \mathrm{n}=504$ \\
\hline Basic $\dagger$ & 0.830 & 0.793 & 0.669 & 0.685 \\
\hline Full $\neq$ & 0.845 & 0.805 & 0.720 & 0.733 \\
\hline
\end{tabular}

* $\mathrm{N}$ indicates sample size and $\mathrm{n}$ indicates the number of $\mathrm{CHD}$ events.

$\uparrow$ Basic model included total cholesterol, HDL cholesterol, systolic blood pressure, antihypertensive medications, current smoking, and diabetes.

‡ Full model included body mass index, waist-hip ratio, Keys score, albumin, residual FEV 1, fibrinogen, factor VII, von Willebrand Factor, lipoprotein(a), heart rate, pack years cigarette smoking, sport activity index, and intima-media thickness. how can the remaining 25 percent be explained? Investigators in the ARIC study examined the improvement in the predictive capability of the basic risk equation by adding the following nontraditional risk factors: body mass index, waist-hip ratio, Keys score, albumin, white blood cell count, "residual" forced expiratory volume at 1 second (FEV1) (calculated as the difference between measured FEV1 and the predicted FEV1 from age, height, and sex), fibrinogen, factor VII, von Willebrand Factor, lipoprotein(a), heart rate, pack years cigarette smoking, sport activity index, and creatinine. ${ }^{4}$ Among the nontraditional risk factors considered, no single factor provided a large improvement in predictive capability of the basic equations only including total cholesterol, HDL cholesterol, systolic blood pressure, antihypertensive medications, current smoking, and diabetes. When nontraditional risk factors and intima-media thickness were added to the basic model, the predictive capacity of the model was significantly improved, as measured by the gain in the AUC above the 0.5. (Table 2)

\section{Have We Reached the Limits in CHD Prediction?}

In the ARIC study, 531 CHD events occurred among 4287 white men during ten years of follow-up period since the baseline examination in $1987 .{ }^{4}$ If we were able to predict CHD perfectly, we could identify those 531 high-risk men at baseline: 100 percent of them should develop CHD events within ten years and none of the remaining 3756 low-risk men would have events. This is theoretically impossible because the CHD risk exists in continuum in a population. Some practical limitations also exist. The CHD events can be unrecognized because of silent myocardial infarction, microinfarction, or unwitnessed deaths. Oftentimes deaths are falsely attributed to CHD. In addition, true CHD risk may relate more closely to lifetime cholesterol level or blood pressure level rather than their levels measured at one point or during a relatively short period. Blood tests may not precisely measure atherogenic or thrombogenic processes. There is always a possibility of unmeasured or undiscovered risk factors as well. Even if the science were complete, there still exists a source of variation from random effects. For these reasons, we will never be able to predict CHD perfectly.

If research since 1979 has improved overall prediction so little, have we discovered all the important risk factors? Is there any evidence that we could predict CHD events better? Some indirect historical evidences suggest that there is more to be discovered. In William Osler's lecture to Royal College of Physicians in 1910, ${ }^{16}$ the number of angina cases per hospital admissions was approximately 1 case per year in large hospitals. In his perspectives, ${ }^{17}$ Paul Dudley White, a graduate of Harvard Medical School in 1911 and founding member of the American Heart Association and International Council of Cardiology, described that there were very few angina cases after review of his voluminous notes 

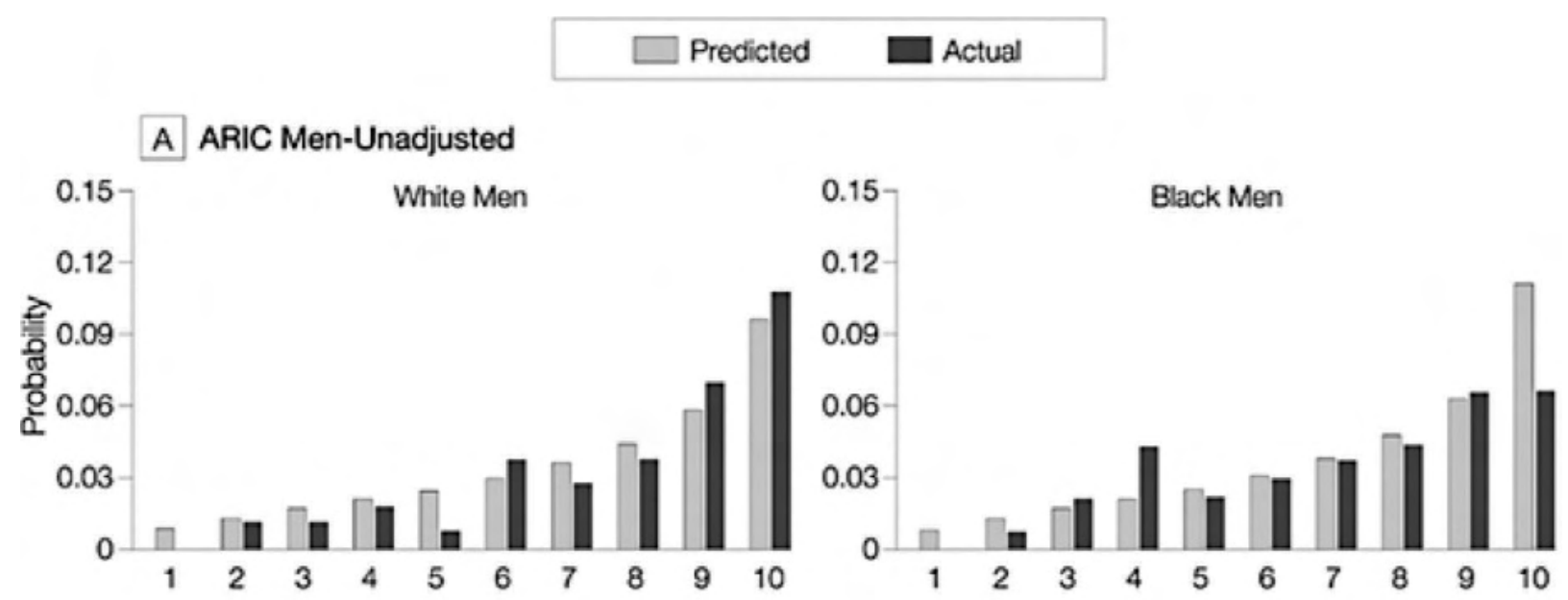

B ARIC Women-Unadjusted
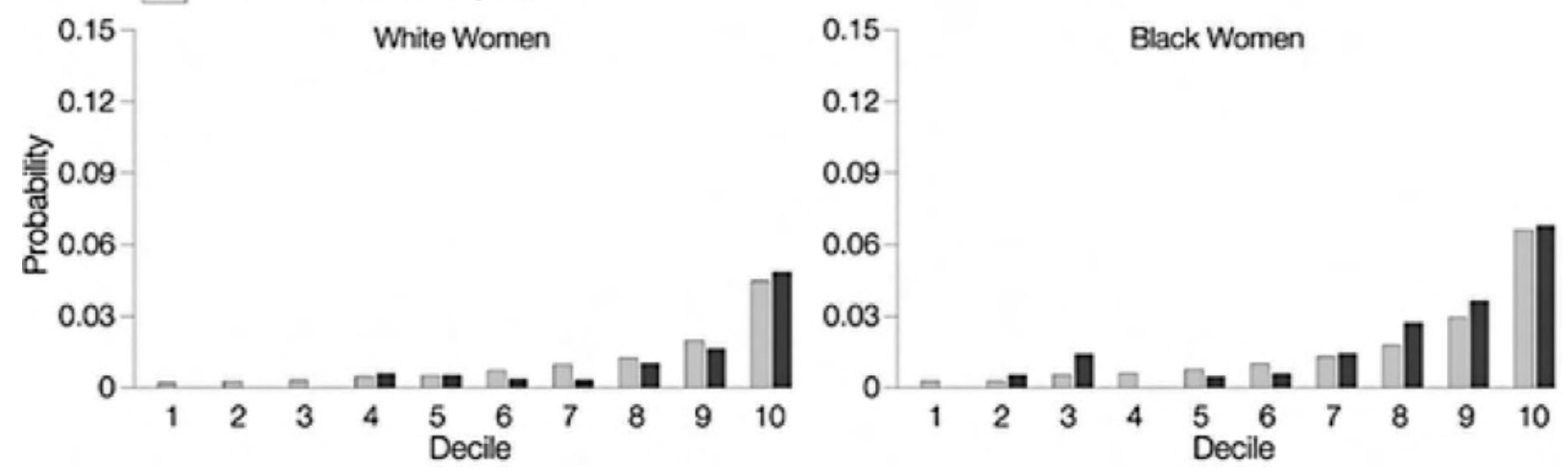

Figure 2. Five-Year Prediction for Hard CHD Events: Performance Measures for Atherosclerosis Risk in Communities Study Men and Women (Adapted from D'Agostino et a $\bar{l}$ )

$X$-axes refer to decile of predicted risk based on the Framingham Heart Study function.

during his medical internship at the Massachusetts General Hospital from 1912 to 1913 . Among his 100 publications in his early career between 1913 and 1926, there were only two papers related to $\mathrm{CHD}$. According to these evidences, $\mathrm{CHD}$ may have been a rare disease in 1910. However, problems exist with historical evidences: they were mainly personal observations and there are major concerns about diagnostic accuracy such as the unavailability of electrocardiogram or cardiac enzyme tests. It is also possible that people did not live long enough to develop CHD.

Besides historical evidences, ecological evidences provide an opportunity to examine the different characteristics and their potential contribution to incident CHD events among populations in different geographic locations. In the International Atherosclerosis Project18 where 21302 autopsies from 15 geographic locations and four race-sex groups were assessed, the age-adjusted percent of intimal surface of coronary arteries involved with raised atherosclerotic lesions varied up to three folds from 6 percent in Durban Bantu or Guatemala to 18 percent in New Orleans whites and Oslo. Prevalence of coronary stenosis among those aged 45 to 54 years also varied from 0 percent in Durban Bantu to 20 percent in New Orleans whites. Another autopsy study compared the prevalence of myocardial infarction between African Americans and Africans in Nigeria and between Asian Americans and Asians in Japan and Korea. ${ }^{19}$ The authors concluded that very low prevalence of myocardial infarction in Africa and Asia was due to environment, not genetics. In a study comparing $\mathrm{CHD}$ incidence in men aged 45 to 64 years who participated in three large National Heart Lung Blood Institutesupported cohort studies in the 1960s, the incidence of CHD in Honolulu Japanese and Puerto Rico men was only 40 percent of that observed in the Framingham Heart Study. ${ }^{20}$ It was suggested that lower levels of risk factors during early lifetime might be responsible for lower CHD incidence. In the Seven Countries Study, ten-year CHD mortality in 16 cohorts was strongly associated with median serum cholesterol levels and the incidence of CHD mortality in low-rate cohorts was 11 percent of that in high-rate cohorts. ${ }^{21}$ Similar findings between serum cholesterol level and CHD mortality were observed in other studies.,22 Estimated CHD reduction effect of lowering cholesterol by $0.6 \mathrm{mmol}(23.2 \mathrm{mg} / \mathrm{dL})$ was 27 percent in cohort studies and $38 \%$ percent in ecological studies. ${ }^{7}$ Like historical evidences, ecologic evidences are not free from limitations: interpretation may be 

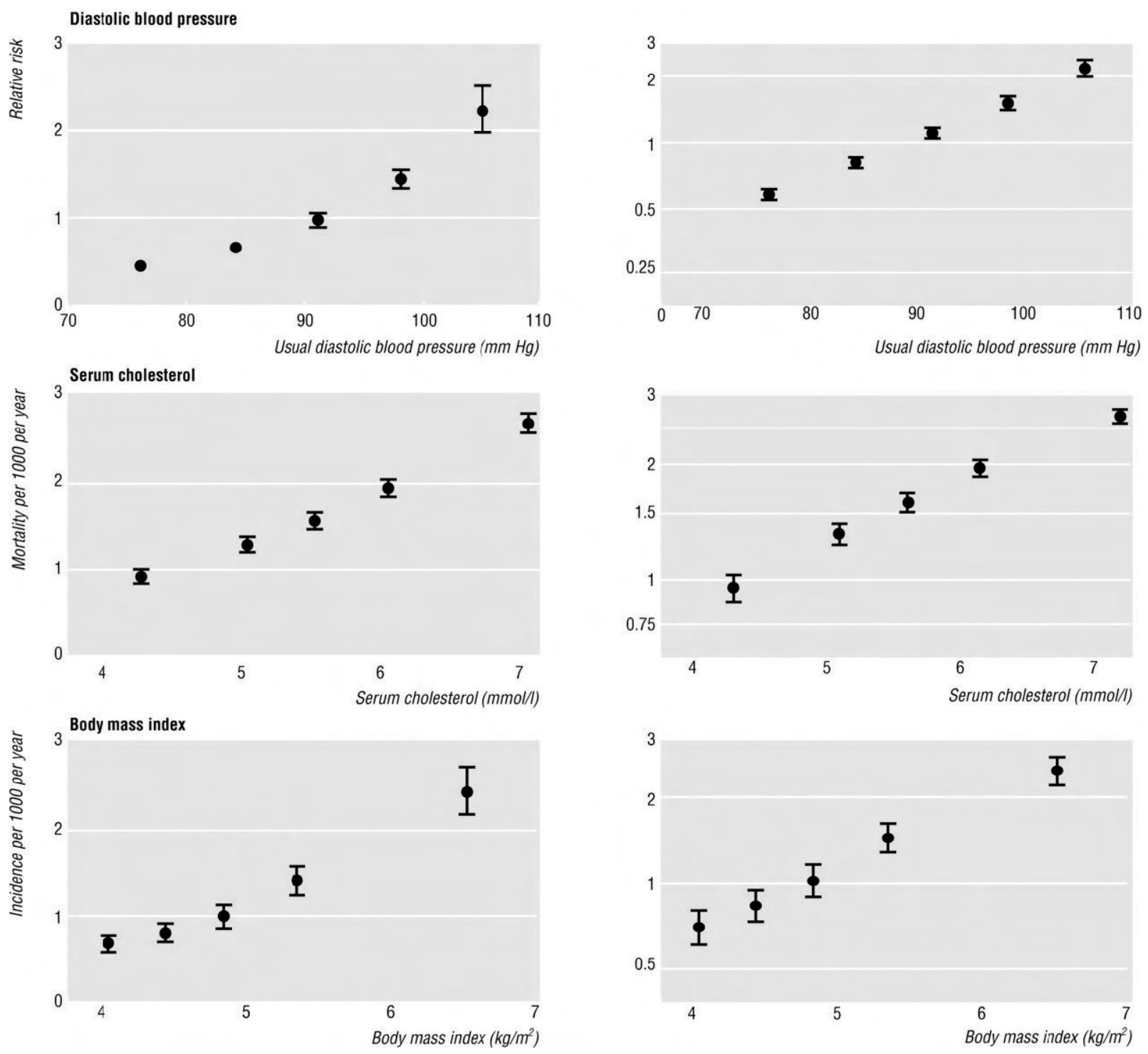

Figure 3. Incidence of CHD According to Diastolic Blood Pressure, Serum Cholesterol, and Body Mass Index (Adapted from Law et al"

Data were obtained from cohort studies. Incidence was plotted on arithmetic scale (left hand plots) and logarithmic scale (right hand plots).

affected by possible genetic difference. In addition, secular changes in many populations have diminished research opportunities. Despite abovementioned limitations, both historical and ecological evidences suggest that some human adult populations have extremely low CHD event rates and provide a unique perspective to better understand the incidence of CHD events.

\section{Is It Possible to Prevent 90 Percent of CHD Events?}

Historical and ecological comparisons have suggested the potential for preventing a large proportion of CHD events in contemporary western countries. ${ }^{7,18,20-24}$ It may be possible to prevent 90 percent of CHD events by reducing standard risk factors to optimal levels. In the MRFIT and CHA studies, young non-smoking men with cholesterol level less than $200 \mathrm{mg} / \mathrm{dL}$ and blood pressure less than or equal to $120 / 80 \mathrm{mmHg}$ had 86 to 92 percent less CHD events than the rest of male participants. ${ }^{6}$ Similar results were observed in the ARIC study. ${ }^{4}$ The benefit from low risk profile was greater for individuals with persisting low risk profile. These studies are prospectively conducted and their estimates are unbiased. Is it feasible for high-risk individuals to reduce their risk to optimal levels? A meta-analysis of risk factor associations with CHD showed that a given change in a risk factor reduces the risk of CHD by a constant proportion of the existing risk regardless of the starting level of the existing risk. ${ }^{25}$ (Figure 3) The authors also emphasized that individuals should be selected for preventive treatment only based on a person's absolute risk level and high-risk individuals should receive interventions to modify all reversible risk factors simultaneously. ${ }^{25}$ With good motivation from high-risk individuals and aggressive strategies against multiple modifiable risk factors, we may be able to achieve large risk reduction. Then, we can ask whether entire populations can reduce their risk to optimal levels. When the exposure to a risk factor is homogeneous within a population, the case-control and cohort methods will fail to detect 
the risk factor as a cause of an outcome. As a result, the approach aiming at high-risk individuals without a population approach will only protect susceptible individuals, but will not control the causes of incidence in the population. ${ }^{26}$ According to population-based studies ${ }^{4,6}$ more than 90 percent has higher than optimal risk levels. People at less than highest risk are less motivated to change their risk levels and interventions have lower benefit to risk ratios. Therefore, a population strategy is essential for substantial effect. ${ }^{26}$ There is also evidence that today's average levels of risk factors should not be considered normal. ${ }^{25}$ When risk factor levels in our ancestors were estimated through studies of isolated communities with a hunter-gatherer lifestyle typical of the Stone Age, the rise in risk factor levels that is currently seen in Western populations did not occur in hunter-gatherer communities and the shift in the Western distributions made current averages high in relation to the prehistoric values. (Table 3) Moreover, lowering all risk factors to optimal levels might not be necessary to prevent the majority of $\mathrm{CHD}$, because even low-risk populations did not have optimal levels of all risk factors. In the Seven Countries Study, the population in Crete had average blood pressure of $134 / 80 \mathrm{mmHg}$ and 57 percent of smokers and the population in Japan had average blood pressure of $130 / 72 \mathrm{mmHg}$ and $75 \%$ of smokers. ${ }^{21}$ It seems that very low cholesterol level itself may suffice.

There are evidences supporting the importance of risk factor levels during early life. The Ni-Hon-San study24 suggested early life risk factors may play "predominant role" and geographical comparisons showed that low serum cholesterol level was associated with lower CHD rate than that estimated from withincountry cohort studies. ${ }^{7,21}$ This emphasizes the need for population strategies to alter lifetime risk factor levels. Lifetime low cholesterol levels can be achieved through early dietary modification such as Mediterranean and low-saturated fat diets.

Prevention of $\mathrm{CHD}$ can be improved by new risk factor discoveries. Major areas of investigation include inflammation, lipoprotein oxidation, vulnerable atherosclerotic plaque, hemostasis, endothelial vasoprotection, and microvascular disease. Even though they improve the CHD prediction only slightly, their preventive potential may be large. In addition, markers of critical processes may be targets of primary or secondary prevention.

\section{Conclusions}

Coronary heart disease is not an inevitable consequence of aging.

Major established risk factors can explain 75 to 90 percent of the incidence of coronary heart disease within populations. If the entire population could reduce known risk factor levels to 1 st decile, up to 90 percent prevention might be achieved.

If persistent low cholesterol levels can be achieved by population strategies affecting childhood, it alone might achieve 90 percent prevention when the children become adults.

Although nontraditional risk factors add little to overall risk prediction, they might provide effective prevention opportunities.

\section{References}

1. Executive Summary of The Third Report of The National Cholesterol Education Program (NCEP) Expert Panel on Detection, Evaluation, And Treatment of High Blood Cholesterol In Adults (Adult Treatment Panel III). JAMA 2001; 285(19):2486-2497.

2. Summary of the second report of the National Cholesterol Education Program (NCEP) Expert Panel on Detection, Evaluation, and Treatment of High Blood Cholesterol in Adults (Adult Treatment Panel II). JAMA 1993; 269(23):3015-3023.

3. The fifth report of the Joint National Committee on Detection, Evaluation, and Treatment of High Blood Pressure (JNC V). Arch Intern Med 1993; 153(2):154-183.

4. Chambless LE, Folsom AR, Sharrett AR et al. Coronary heart disease risk prediction in the Atherosclerosis Risk in Communities (ARIC) study. J Clin Epidemiol 2003; 56(9):880-890.

5. D'Agostino RB, Sr., Grundy S, Sullivan LM, Wilson P. Validation of the Framingham coronary heart disease prediction scores: results of a multiple ethnic groups investigation. JAMA 2001; 286(2):180-187.

6. Stamler J, Stamler R, Neaton JD et al. Low risk-factor profile and long-term cardiovascular and noncardiovascular mortality and life expectancy: findings for 5 large cohorts of young adult and middle-aged men and women. JAMA 1999; 282(21):2012-2018.

7. Law MR, Wald NJ. An ecological study of serum cholesterol and ischaemic heart disease between 1950 and 1990. Eur J Clin Nutr 1994; 48(5):305-325.

8. Wilson PW, D'Agostino RB, Levy D, Belanger AM, Silbershatz H, Kannel WB. Prediction of coronary heart disease using risk factor categories. Circulation 1998; 97(18):1837-1847.

9. Gordon T, Kannel WB. Multiple risk functions for predicting coronary heart disease: the concept, accuracy, and application. Am Heart J 1982; 103(6):1031-1039.

10. Kannel WB, McGee D, Gordon T. A general cardiovascular risk profile: the Framingham Study. Am J Cardiol 1976; 38(1):46-51.

11. Nam B-H. Discrimination and Calibration in Survival Analysis [dissertation] Boston, MA: Boston University; 2000.

12. Sackett D, Haynes R, Guyatt G, Tugwell P. Clinical Epidemiology: A Basic Science for Clinical Medicine, 2nd Edition. Philadelphia, PA: Lippincott Williams \& Wilkins; 1991.

13. Keys A, Aravanis C, Blackburn $\mathrm{H}$ et al. Coronary heart disease: overweight and obesity as risk factors. Ann Intern Med 1972; 77(1):15-27.

14. Leaverton PE, Sorlie PD, Kleinman JC et al. Representativeness of the Framingham risk model for coronary heart disease mortality: a comparison with a national cohort study. J Chronic Dis 1987; 40(8):775-784.

15. Magnus P, Beaglehole R. The real contribution of the major risk factors to the coronary epidemics: time to end the "only-50\%" myth. Arch Intern Med 2001; 161(22):2657-2660

16. Osler W. The Lumleian lectures on angina pectoris. Lancet 1910; 1:697-701.

17. White PD. Perspectives. Prog Cardiovasc Dis 1971; 14(3):250-255.

18. Tejada C, Strong JP, Montenegro MR, Restrepo C, Solberg LA. Distribution of coronary and aortic atherosclerosis by geographic location, race, and sex. Lab Invest 1968; 18(5):509-526.

19. Lee KT, Nail R, Sherman LA et al. Geographic Pathology of Myocardial Infarction. Am J Cardiol 1964; 13:30-40.

20. Gordon T, Garcia-Palmieri MR, Kagan A, Kannel WB, Schiffman J. Differences in coronary heart disease in Framingham, Honolulu and Puerto Rico. J Chronic Dis 1974; 27(7-8):329-344.

21. Keys A. Coronary heart disease, serum cholesterol, and the diet. Acta Med Scand 1980; 207(3):153-160.

22. Chen Z, Peto R, Collins R, MacMahon S, Lu J, Li W. Serum cholesterol concentration and coronary heart disease in population with low cholesterol concentrations. BMJ 1991; 303(6797):276-282.

23. Robertson TL, Kato H, Rhoads GG et al. Epidemiologic studies of coronary heart disease and stroke in Japanese men living in Japan, Hawaii and California. Incidence of myocardia infarction and death from coronary heart disease. Am J Cardiol 1977; 39(2):239-243.

24. Robertson TL, Kato H, Gordon T et al. Epidemiologic studies of coronary heart disease and stroke in Japanese men living in Japan, Hawaii and California. Coronary heart disease risk factors in Japan and Hawaii. Am J Cardiol 1977; 39(2):244-249.

25. Law MR, Wald NJ. Risk factor thresholds: their existence under scrutiny. BMJ 2002; 324(7353):1570-1576.

26. Rose G. Sick individuals and sick populations. Int J Epidemiol 1985; 14(1):32-38 